\title{
Confiabilidade das classificações artroscópicas das lesões condrais do quadril*
}

\section{Reliability of the Arthroscopic Classifications of Hip Chondral Lesions}

\author{
Antônio Augusto Guimarães Barros ${ }^{1}$ (1) Carlos César Vassalo ${ }^{1} \quad$ Lincoln Paiva Costa ${ }^{1}$ \\ Juan Gómez-Hoyos ${ }^{2}$ Vinícius de Oliveira Paganini ${ }^{1}$ Marco Antônio Percope de Andrade ${ }^{3}$ \\ 1 Hospital Madre Teresa, Belo Horizonte, MG, Brasil \\ ${ }^{2}$ Centro de Preservação do Quadril, Baylor Scott and White Research \\ Institute, Dallas, TX, EUA \\ ${ }^{3}$ Departamento do Aparelho Locomotor, Faculdade de Medicina, \\ Universidade Federal de Minas Gerais, Belo Horizonte, MG, Brasil \\ Endereço para correspondência Antônio Augusto Guimarães Barros, \\ Master, Hospital Madre Teresa, Belo Horizonte, MG, Brasil \\ (e-mail: antonioagbarros@gmail.com). \\ Rev Bras Ortop 2019;54:440-446.
}

\section{Resumo \\ Palavras-chave \\ - artroscopia \\ - quadril \\ - doenças das cartilagens/ classificação \\ - reprodutibilidade dos testes}

\section{Abstract}

Objetivo Avaliar a confiabilidade inter- e intraobservador das classificações de Outerbridge, Beck e Haddad para lesões da cartilagem articular acetabular com o uso da via artroscópica.

Métodos Foram avaliados 60 vídeos de artroscopias do quadril por 4 cirurgiões em 2 momentos para avaliar a reprodutibilidade inter- e intraobservador das classificações. Os dados foram analisados a partir do cálculo do índice Kappa de Cohen ponderado. Resultados Os valores do Kappa ponderado médio na avaliação interobservador das classificações de Outerbridge, Beck e Haddad foram, respectivamente, 0,72, 0,78 e 0,68 . As três classificações foram consideradas como de boa concordância interobservador. Com relação à avaliação intraobservador das classificações de Outerbridge, Beck e Haddad, os valores Kappa foram, respectivamente, 0,9, 0,9 e 0,93. As três classificações foram consideradas excelentes na comparação intraobservador. Conclusão Na presente série, as classificações de Outerbridge, Beck e Haddad apresentaram boa reprodutibilidade interobservador e excelente reprodutibilidade intraobservador ao avaliar lesões condrais acetabulares por via artroscópica.

Objective To evaluate the inter- and intraobserver reliability of the Outerbridge, Beck, and Haddad classifications for acetabular joint cartilage lesions through the arthroscopic procedure.

Methods A total of 60 hip arthroscopy videos were evaluated twice by 4 surgeons at 2 different times to assess the inter- and intraobserver reproducibility of the classifications, and the data was analyzed by means of the weighted Cohen Kappa index.

(D)Antônio Augusto Guimarães Barros's ORCID is https://orcid.org/ 0000-0003-3701-1937.

recebido

12 de Novembro de 2017

aceito

09 de Janeiro de 2018
DOI https://doi.org/

10.1016/j.rbo.2018.01.002. ISSN $0102-3616$.
Copyright $\odot 2019$ by Sociedade Brasileira License terms de Ortopedia e Traumatologia. Published by Thieme Revnter Publicações Ltda, Rio de Janeiro, Brazil 
Keywords

- arthroscopy

- hip

- cartilage diseases/ classification

- reproducibility of results
Results The mean weighted Kappa values in the interobserver assessment of the Outerbridge, Beck, and Haddad classifications were, respectively, 0.72, 0.78, and 0.68. The three classifications were considered as presenting good interobserver agreement. Regarding the intraobserver assessment of the Outerbridge, Beck, and Haddad classifications, the weighted Kappa values were, respectively, 0.9, 0.9, and 0.93. The three classifications were considered as presenting excellent intraobserver agreement. Conclusion In the present series, the Outerbridge, Beck, and Haddad classifications presented good interobserver reproducibility and excellent intraobserver reproducibility when evaluating acetabular chondral lesions by the arthroscopic approach.

\section{Introdução}

A artroscopia do quadril surgiu como um método minimamente invasivo para o tratamento e diagnóstico de diversas doenças intra- e extra-articulares do quadril. ${ }^{1}$ Essa via de acesso fornece excelente visualização da superfície articular e permite que o cirurgião identifique estágios precoces de dano cartilaginoso. ${ }^{2}$ As lesões da cartilagem articular do quadril podem ser causadas principalmente por trauma, artrites inflamatórias, displasia acetabular ou impacto femoroacetabular (IFA). ${ }^{3}$

Após tratar jovens adultos com o diagnóstico de artrose idiopática do quadril, Ganz et $\mathrm{al}^{4}{ }^{4}$ perceberam que certas características morfológicas dessa articulação resultavam de contato anormal entre o fêmur proximal e o rebordo acetabular, o chamado IFA. Posteriormente, foi observado que esse contato anormal leva a padrões característicos de dano do labrum acetabular e da cartilagem adjacente. Lesão labral anterossuperior juntamente com o dano cartilaginoso posteroinferior por contragolpe na cabeça femoral e na superfície acetabular são associados ao impacto tipo Pincer. A separação do labrum anterossuperior do rebordo acetabular e o cisalhamento ou a delaminação da cartilagem articular acetabular adjacente são mais indicativos do impacto tipo CAM. ${ }^{5}$ Comumente, os pacientes com IFA apresentam a associação das duas deformidades. Estudos recentes apontam para uma forte associação entre a osteoartrite (OA) do quadril e o IFA. ${ }^{6}$ Por isso, atualmente, há grande interesse no tratamento das lesões labrais e condrais no quadril com o objetivo de avaliar se essas intervenções serão capazes de retardar ou até mesmo de evitar o desenvolvimento da $\mathrm{OA}$ do quadril nesses pacientes.

Com o rápido crescimento do diagnóstico do IFA e da artroscopia do quadril como via de acesso para o tratamento, houve também um crescimento exponencial das publicações relacionadas a esse assunto. ${ }^{7}$ Porém, há uma grande variabilidade na literatura a respeito das indicações, dos resultados, dos métodos de avaliação, do manuseio da cápsula articular e das partes moles, assim como da reabilitação. Parte dessa variabilidade está associada à comparação de pacientes com lesões labrais e condrais em estágios e gravidade diferentes. Com o objetivo de reduzir essa variabilidade, deve-se comparar pacientes com lesões classificadas em estágios de gravidade semelhantes.

Os sistemas de classificação ajudam na comunicação, no planejamento do tratamento e na avaliação do prognóstico.
Um sistema ideal de classificação deve ser de fácil aplicabilidade, confiável, reprodutível, incluir todo espectro de lesões, ser mutuamente excludente, lógico, e clinicamente útil. ${ }^{8}$

O objetivo do presente estudo é avaliar a confiabilidade inter- e intraobservador das classificações das lesões da cartilagem articular do quadril através da via artroscópica e, com isso, definir quais são as classificações de maior reprodutibilidade a partir do cálculo do índice Kappa de Cohen ponderado. Com o presente estudo, espera-se encontrar as classificações por via artroscópica que apresentam maior reprodutibilidade para que a comunicação, o resultado do tratamento e a avaliação do prognóstico sejam feitos de modo confiável. A hipótese é que as três classificações estudadas tenham moderada confiabilidade inter- e intraobservador.

\section{Materiais e Métodos}

A partir de uma base de dados de 278 vídeos de artroscopia do quadril, com gravação previamente autorizada pelo paciente, foram selecionados inicialmente 70 vídeos. Estes pacientes foram convidados a participar do estudo e a assinar um termo de consentimento. 0 critério de inclusão consistiu de todos os pacientes submetidos a artroscopia do quadril para IFA. Os critérios de exclusão incluíram presença de artrose radiológica (Tonnis $>2$ ), ${ }^{9}$ história de cirurgia prévia no quadril (aberta ou artroscópica), vídeos com imagem de má qualidade, visualização inadequada, palpação inadequada das lesões, e recusa a participar do estudo. Após aplicados os critérios acima, a amostra final foi de 60 vídeos.

As operações foram feitas por 2 cirurgiões experientes em artroscopia do quadril ( $>200$ procedimentos feitos por cada cirurgião) e seguiram um padrão já usado rotineiramente por ambos os cirurgiões. As artroscopias foram feitas com o paciente em posição supina na mesa de tração com o uso dos portais anterolateral e medioanterior. A avaliação intraarticular do acetábulo foi feita com a câmera no portal anterolateral e, com um instrumento de palpação no acesso medioanterior, foi feita a inspeção da superfície articular, do labrum e da junção condrolabral.

Um dos autores, cirurgião de quadril treinado em artroscopia, o qual não participou das cirurgias, reviu cada gravação e as editou. Após a edição, cada gravação teve duração de $\sim 1$ minuto e incluiu a inspeção da superfície articular, do labrum e da junção condrolabral. Este processo resultou em 60 gravações digitais de artroscopias do quadril. 
Quatro cirurgiões de quadril com experiência em artroscopia do quadril (todos com pelo menos 2 anos de experiência e participação em $>150$ casos de artroscopia de quadril) participaram do estudo. Durante uma reunião, os quatro cirurgiões discutiram os esquemas de classificação descritos para as lesões da cartilagem articular: Outerbridge, ${ }^{10}$ Beck et a ${ }^{11} \mathrm{e}$ Konan et $\mathrm{al}^{12}$ (-Tabela 1). Após 15 dias, houve um novo encontro para a prática do uso das classificações em vídeos não incluídos no presente estudo. Após o treinamento dos médicos, cada cirurgião, separadamente, classificou as lesões encontradas nos vídeos de acordo com as classificações propostas acima. Se $>1$ achado fosse encontrado, os revisores foram instruídos a relatar apenas o achado mais grave. Os valores iniciais de cada revisor foram usados para a análise interobservador. Em um segundo momento, 1 mês depois, foi solicitado aos revisores que as lesões dos mesmos vídeos fossem novamente classificadas em ordem diferente. Esses valores foram pareados por caso e revisor e foram comparados com os primeiros resultados para uma análise intraobservador.

A avaliação estatística da confiabilidade das classificações incluiu a porcentagem de concordância e os valores ponderados de Kappa propostos por Cohen. ${ }^{13}$ Os valores Kappa foram classificados como descrito por Landis et al: ${ }^{14}$ entre 0,81 e 1,0 indicaram excelente concordância; de 0,61 a 0,80 , boa concordância; de 0,41 a 0,60, moderada concordância; de 0,21 a 0,40, razoável concordância; e de 0 a 0,20, fraca concordância. As análises estatísticas foram feitas nos programas $\mathrm{R}$ versão 3.3.2 e Minitab versão 18 (Minitab, LLC, State College, PA, USA).
A pesquisa foi previamente aprovada pelo Comitê de Ética em Pesquisa e feita de acordo com a Declaração de Helsinque da Associação Médica Mundial.

\section{Resultados}

As características demográficas dos participantes do estudo estão resumidas na - Tabela $\mathbf{2}$. A análise descritiva da classificação das lesões por cada observador com os sistemas de Outerbridge, Beck e Haddad observados no primeiro momento é apresentada na - Tabela 3. Esses resultados são ilustrados nas - Figs. 1, 2, 3. Com esses dados, ao se avaliar a concordância interobservador, observa-se que, em um caso, a concordância da classificação de Outerbridge foi moderada (I e IV), em quatro foi boa (I e II; I e III; II e IV; III e IV), e em um

Tabela 2 Características demográficas dos participantes

\begin{tabular}{|c|c|}
\hline \multicolumn{2}{|l|}{ Gênero, n (\%) } \\
\hline Masculino & $32(53 \%)$ \\
\hline Feminino & $28(47 \%)$ \\
\hline Idade, média (desvio padrão) & 33 anos $( \pm 7)$ \\
\hline \multicolumn{2}{|l|}{ Deformidade, $n$ (\%) } \\
\hline Combinada (CAM e Pincer) & $31(52 \%)$ \\
\hline CAM & $27(45 \%)$ \\
\hline Pincer & $2(3 \%)$ \\
\hline
\end{tabular}

Tabela 1 Classificações de Outerbridge, Beck e Haddad

\begin{tabular}{|c|c|}
\hline Tipos & Descrição \\
\hline \multicolumn{2}{|c|}{ Outerbridge } \\
\hline 0 & Cartilagem sem alterações macroscópicas \\
\hline 1 & Amolecimento ou edema \\
\hline 2 & Fragmentação/lesão < 1,3cm \\
\hline 3 & Fragmentação/lesão $>1,3 \mathrm{~cm}$ \\
\hline 4 & Exposição óssea \\
\hline \multicolumn{2}{|l|}{ Beck } \\
\hline 0 & Cartilagem sem alterações macroscópicas \\
\hline 1 & Malacia; Rugosidade da superfície, fibrilação \\
\hline 2 & $\begin{array}{l}\text { Destacamento; Perda de fixação ao osso subcondral, cartilagem } \\
\text { macroscopicamente sólida, fenômeno de carpete }\end{array}$ \\
\hline 3 & $\begin{array}{l}\text { Clivagem; Perda de fixação ao osso subcondral, bordas } \\
\text { desgastadas, adelgaçamento da cartilagem, flap }\end{array}$ \\
\hline 4 & Defeito de espessura total \\
\hline \multicolumn{2}{|c|}{ Haddad } \\
\hline 0 & Cartilagem sem alterações macroscópicas \\
\hline 1 & Sinal de onda; Perda de fixacão do osso subcondral \\
\hline 2 & $\begin{array}{l}\text { Clivagem; Óbvia separação na juncão condrolabral, mas a sondagem } \\
\text { artroscópica da lesão mostra aderência da cartilagem articular ao } \\
\text { osso subjacente sem evidência de delaminação }\end{array}$ \\
\hline 3 & Delaminação; Destacamento macroscópico da cartilagem do osso acetabular \\
\hline 4 & Osso exposto \\
\hline
\end{tabular}


Tabela 3 Avaliação das três classificações para as lesões da cartilagem no primeiro momento avaliado

\begin{tabular}{|c|c|c|c|c|c|c|c|c|}
\hline \multicolumn{3}{|c|}{ Classificações } & \multicolumn{6}{|c|}{ Observador } \\
\hline & \multicolumn{2}{|l|}{$I$} & \multicolumn{2}{|l|}{ II } & \multicolumn{2}{|l|}{ III } & \multicolumn{2}{|l|}{ IV } \\
\hline & $n$ & $\%$ & $n$ & $\%$ & $n$ & $\%$ & $n$ & $\%$ \\
\hline \multicolumn{9}{|c|}{ Outerbridge } \\
\hline 0 & 8 & 13,33 & 8 & 13,33 & 5 & 8,33 & 19 & 31,67 \\
\hline 1 & 25 & 41,67 & 28 & 46,67 & 30 & 50,00 & 16 & 26,67 \\
\hline 2 & 11 & 18,33 & 13 & 21,67 & 8 & 13,33 & 15 & 25,00 \\
\hline 3 & 8 & 13,33 & 7 & 11,67 & 11 & 18,33 & 2 & 3,33 \\
\hline 4 & 8 & 13,33 & 4 & 6,67 & 6 & 10,00 & 8 & 13,33 \\
\hline \multicolumn{9}{|c|}{ Beck } \\
\hline 0 & 8 & 13,33 & 6 & 10,00 & 7 & 11,67 & 14 & 23,33 \\
\hline 1 & 22 & 36,67 & 13 & 21,67 & 12 & 20,00 & 8 & 13,33 \\
\hline 2 & 11 & 18,33 & 19 & 31,67 & 21 & 35,00 & 15 & 25,00 \\
\hline 3 & 10 & 16,67 & 16 & 26,67 & 14 & 23,33 & 14 & 23,33 \\
\hline 4 & 9 & 15,00 & 6 & 10,00 & 6 & 10,00 & 9 & 15,00 \\
\hline \multicolumn{9}{|c|}{ Haddad } \\
\hline 0 & 10 & 16,67 & 10 & 16,67 & 13 & 21,67 & 15 & 25,00 \\
\hline 1 & 22 & 36,67 & 15 & 25,00 & 18 & 30,00 & 5 & 8,33 \\
\hline 2 & 10 & 16,67 & 13 & 21,67 & 9 & 15,00 & 17 & 28,33 \\
\hline 3 & 10 & 16,67 & 16 & 26,67 & 13 & 21,67 & 15 & 25,00 \\
\hline 4 & 8 & 13,33 & 6 & 10,00 & 7 & 11,67 & 8 & 13,33 \\
\hline
\end{tabular}

n, número de observações; \%, porcentagem.

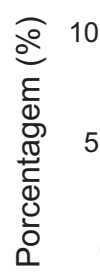

50

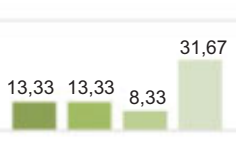

Grau 0

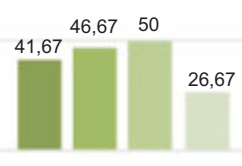

Grau 1

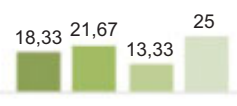

Grau 2

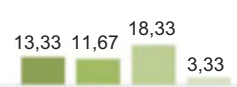

Grau 3

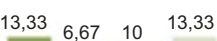

Grau 4

$=|=I I=I I|=I V$

Fig. 1 Frequência das lesões da cartilagem, segundo os quatro observadores, com a classificação de Outerbridge.

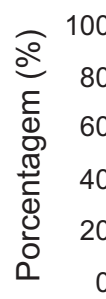

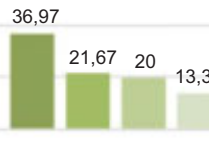

Malácia

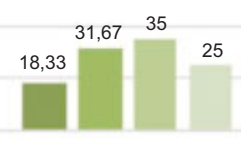

Descolamento

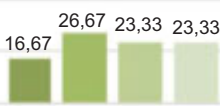

Clivagem

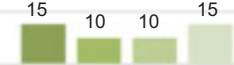

Defeito

EI $=$ II $=$ III

Fig. 2 Frequência das lesões da cartilagem, segundo os quatro observadores, com a classificação de Beck. 


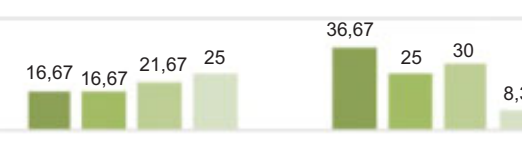

Grau 0
Grau 1

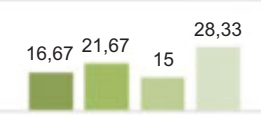

Grau 2

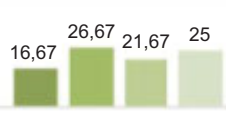

Grau 3

\section{$\begin{array}{llll}13,33 & 10 \quad 11,67 & 13,33\end{array}$}

Grau 4

$$
=\mathrm{I}=\mathrm{II}=\mathrm{III}=\mathrm{IV}
$$

Fig. 3 Frequência das lesões da cartilagem, segundo os quatro observadores, com a classificação de Haddad.

foi excelente (II e III). Os Kappa variaram entre 0,58 e 0,82, com um valor médio de 0,72 , ou seja, a classificação média entre os observadores foi considerada boa.

Com relação à classificação de Beck, conclui-se que em quatro casos a concordância foi boa (I e II; I e III; I e IV; II e IV) e em dois foi excelente (II e III; III e IV). Os Kappa variaram entre 0,74 e 0,84 , com um valor médio de 0,78 , ou seja, a classificação média entre os observadores foi considerada boa.

Os resultados da classificação de Haddad para a concordância interobservador foram moderados em um caso (III e IV), e foram bons em cinco (I e II; I e III; I e IV; II e III; II e IV). Os Kappa variaram entre 0,60 e 0,78, com um valor médio de 0,68 , ou seja, a classificação média entre os observadores foi considerada boa. A - Tabela 4 apresenta o resumo da avaliação interobservador.

Com relação à avaliação intraobservador, a classificação de Outerbridge apresentou valores Kappa entre 0,83 e 0,94, com um valor médio de 0,90 , ou seja, a classificação média entre os observadores foi considerada excelente. Os quatro observadores tiveram concordância da classificação Beck para as lesões da cartilagem classificada como excelente. Os Kappa variaram entre 0,87 e 0,92, com um valor médio de 0,90 , ou seja, a classificação média entre os observadores foi considerada excelente. Da mesma forma, a classificação Haddad foi considerada excelente, com valores de Kappa entre 0,89 e 0,97, com um valor médio de 0,93. A - Tabela 5 apresenta o resumo da avaliação intraobservador.

Tabela 4 Avaliação interobservador com o Kappa ponderado

\begin{tabular}{|c|c|c|c|c|c|}
\hline Classificação & Kappa & Classificação do Kappa & IC95\% & Taxa de concordância & IC95\% \\
\hline Outerbridge & 0,72 & Boa & & & \\
\hline Observador I e II & 0,73 & Boa & $0,53-0,94$ & 0,62 & $0,48-0,74$ \\
\hline Observador I e III & 0,72 & Boa & $0,52-0,92$ & 0,58 & $0,45-0,71$ \\
\hline Observador I e IV & 0,58 & Moderada & $0,37-0,80$ & 0,43 & $0,31-0,57$ \\
\hline Observador II e III & 0,82 & Excelente & $0,72-0,89$ & 0,63 & $0,50-0,75$ \\
\hline Observador II e IV & 0,72 & Boa & $0,60-0,87$ & 0,52 & $0,39-0,65$ \\
\hline Observador III e IV & 0,73 & Boa & $0,58-0,83$ & 0,43 & $0,31-0,57$ \\
\hline Beck & 0,78 & Boa & & & \\
\hline Observador I e II & 0,77 & Boa & $0,64-0,90$ & 0,57 & $0,43-0,69$ \\
\hline Observador I e III & 0,74 & Boa & $0,59-0,90$ & 0,53 & $0,40-0,66$ \\
\hline Observador I e IV & 0,74 & Boa & $0,62-0,86$ & 0,50 & $0,37-0,63$ \\
\hline Observador II e III & 0,84 & Excelente & $0,77-0,91$ & 0,68 & $0,55-0,79$ \\
\hline Observador II e IV & 0,78 & Boa & $0,66-0,91$ & 0,62 & $0,48-0,74$ \\
\hline Observador III e IV & 0,82 & Excelente & $0,81-0,84$ & 0,63 & $0,50-0,75$ \\
\hline Haddad & 0,68 & Boa & & & \\
\hline Observador I e II & 0,68 & Boa & $0,48-0,88$ & 0,50 & $0,37-0,63$ \\
\hline Observador I e III & 0,78 & Boa & $0,66-0,90$ & 0,60 & $0,47-0,72$ \\
\hline Observador I e IV & 0,61 & Boa & $0,39-0,82$ & 0,42 & $0,29-0,55$ \\
\hline Observador II e III & 0,75 & Boa & $0,54-0,96$ & 0,67 & $0,53-0,78$ \\
\hline Observador II e IV & 0,66 & Boa & $0,45-0,86$ & 0,50 & $0,37-0,63$ \\
\hline Observador III e IV & 0,60 & Moderada & $0,37-0,82$ & 0,43 & $0,31-0,57$ \\
\hline
\end{tabular}

Abreviação: IC, intervalo de confiança. 
Tabela 5 Avaliação intraobservador com o Kappa ponderado

\begin{tabular}{|l|l|l|l|l|l|}
\hline Classificação & Kappa & Classificação do Kappa & IC95\% & Taxa de concordância & IC95\% \\
\hline Outerbridge & $\mathbf{0} 90$ & Excelente & & & $0,58-0,82$ \\
\hline Observador I & 0,83 & Excelente & $0,70-0,96$ & 0,72 & $0,60-0,84$ \\
\hline Observador II & 0,90 & Excelente & $0,84-0,94$ & 0,73 & $0,71-0,91$ \\
\hline Observador III & 0,94 & Excelente & $0,89-0,96$ & 0,83 & $0,65-0,88$ \\
\hline Observador IV & 0,91 & Excelente & $0,82-0,96$ & 0,78 & \\
\hline Beck & $\mathbf{0 , 9 0}$ & Excelente & & & \\
\hline Observador I & 0,90 & Excelente & $0,83-0,95$ & 0,77 & $0,64-0,86$ \\
\hline Observador II & 0,92 & Excelente & $0,79-1,00$ & 0,87 & $0,75-0,94$ \\
\hline Observador III & 0,87 & Excelente & $0,70-1,00$ & 0,80 & $0,67-0,89$ \\
\hline Observador IV & 0,91 & Excelente & $0,80-1,00$ & 0,87 & $0,75-0,94$ \\
\hline Haddad & $\mathbf{0 , 9 3}$ & Excelente & & & \\
\hline Observador I & 0,93 & Excelente & $0,87-0,97$ & 0,80 & \\
\hline Observador II & 0,89 & Excelente & $0,75-1,00$ & 0,85 & \\
\hline Observador III & 0,94 & Excelente & $0,88-0,98$ & 0,85 & $0,67-0,89$ \\
\hline Observador IV & 0,97 & Excelente & $0,94-0,99$ & 0,88 & $0,73-0,93$ \\
\hline
\end{tabular}

Abreviação: IC, intervalo de confiança.

\section{Discussão}

O presente estudo mostrou que as classificações para avaliação das lesões condrais do acetábulo por via artroscópica são reprodutíveis tanto na avaliação inter- quanto intraobservador. Na avaliação interobservador, por meio do valor do coeficiente Kappa ponderado, foi observada boa concordância nas três classificações estudadas, e na avaliação intraobservador o coeficiente Kappa ponderado foi considerado excelente em todas as classificações avaliadas.

Os estudos que avaliaram a reprodutibilidade das classificações das lesões condrais no quadril mostram resultados com grande variação. Amenabar et $\mathrm{al}^{3}{ }^{3}$ ao avaliarem a reprodutibilidade das classificações de Outerbridge, Beck e Haddad no diagnóstico das lesões condrais por via artroscópica, encontraram um valor Kappa interobservador de 0,28, 0,33 e 0,47, respectivamente. A classificação de Outerbridge foi considerada de fraca concordância, e as classificações de Beck e Haddad foram consideradas como de moderada concordância. Os valores Kappa intraobservador foram de 0,62, 0,63 e 0,68 para as classificações de Outerbridge, Beck e Haddad, respectivamente; todas apresentaram moderada concordância. No presente estudos, os valores do coeficiente Kappa ponderado encontrados foram maiores, o que pode ser explicado parcialmente pela presença de duas reuniões prévias para discussão e treinamento das classificações. Além disso, Amenabar et $a^{3}$ fizeram a segunda rodada de classificações 4 meses após a primeira. No presente estudo, o intervalo entre as rodadas de classificação foi de apenas 1 mês; talvez, se os cirurgiões as classificassem novamente após um intervalo de tempo maior, a reprodutibilidade intraobservador tivesse sido menor.

Em um estudo retrospectivo que envolveu 40 vídeos e 4 observadores, Nepple et al $^{15}$ avaliaram a reprodutibilidade da classificação de Beck para as lesões labrais e condrais do acetábulo. Com relação às lesões condrais, foram encontrados valores Kappa médios de 0,65 (de 0,49 a 0,74) e de 0,80 (de 0,68 a 0,86 ) para a reprodutibilidade interobservador e intraobservador, respectivamente. Assim como no presente estudo, a classificação de Beck interobservador foi considerada como de boa concordância, mas a reprodutibilidade intraobservador foi boa, e não excelente, como no presente estudo. No presente estudo, a classificação de Beck mostrou-se como a de maior reprodutibilidade interobservador, com um valor Kappa médio de 0,78 . Ao estudar a classificação de Haddad, Konan et al ${ }^{12}$ observaram uma alta reprodutibilidade com coeficiente de correlação intraclasse de 0,88 na avaliação interobservador, e de 0,81 na avaliação intraobservador. No presente estudo, a classificação de Haddad apresentou a maior reprodutibilidade intraobservador, com um valor Kappa médio de 0,93.

Lasmar et al $^{16}$ avaliaram a reprodutibilidade da classificação de Outerbridge para as lesões condrais no joelho diagnosticadas por via artroscópica, e foi encontrado um valor Kappa interobservador de 0,43 , e intraobservador de 0,31 , indicando concordância moderada. Porém, entre os seis observadores, foram incluídos dois residentes, e em outros dois observadores não ficou clara a experiência em cirurgia do joelho. A presença de um valor Kappa interobservador maior do que o intraobservador se mostrou inesperada. No presente estudo, todos os observadores são cirurgiões de quadril com experiência em procedimentos artroscópicos, o que teoricamente aumenta a probabilidade de concordância entre os observadores. Em outro estudo que também avaliou a reprodutibilidade da classificação de Outerbridge para lesões no joelho, Cameron et al $^{17}$ encontraram um Kappa interobservador de 0,72, e intraobservador de 0,91 entre médicos com $>5$ anos de prática; valores bem próximos dos encontrados no presente estudo, o qual também incluiu como observadores apenas cirurgiões experientes em artroscopia do quadril.

O presente estudo mostra que as classificações de Outerbridge, Beck e Haddad são reprodutíveis o bastante para serem 


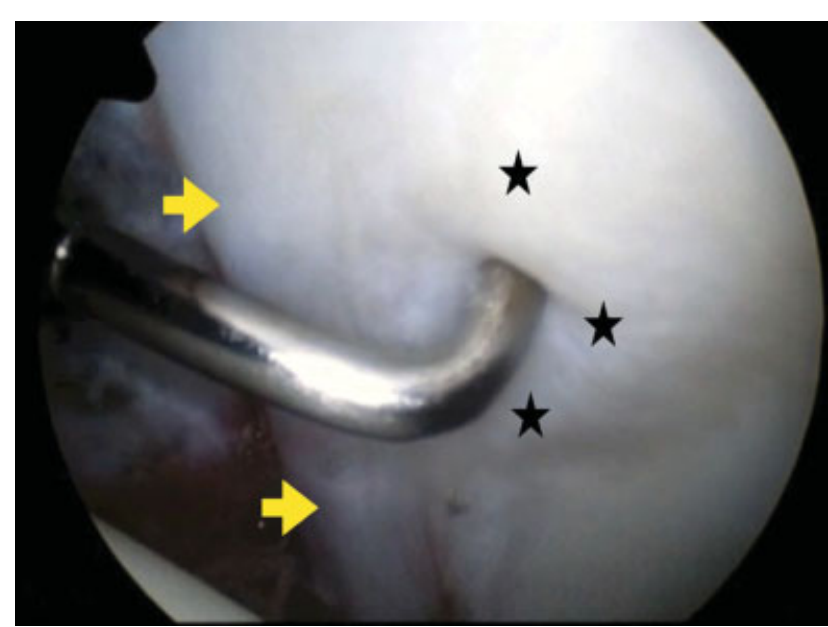

Fig. 4 Imagem artroscópica do quadril indica lesão da cartilagem acetabular através do "sinal da onda" (estrelas) e do labrum acetabular (seta amarela)

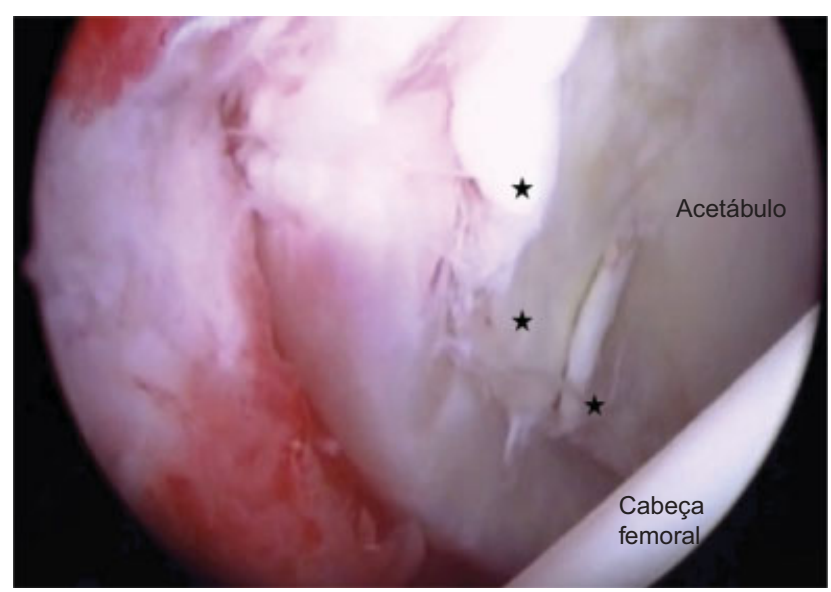

Fig. 5 Imagem artroscópica do quadril indica lesão da cartilagem acetabular com formação de flaps (estrelas).

aplicadas na prática clínica. Porém, além da reprodutibilidade, uma classificação deve descrever de modo adequado as lesões e oferecer orientação para o tratamento e o prognóstico. A classificação de Outerbridge não foi desenvolvida para avaliar as lesões condrais do acetábulo e não leva em consideração lesões características produzidas pelo IFA, como o sinal da onda (-Fig. 4) ou a presença de destacamento da cartilagem com formação de flap (- Fig. 5). Portanto, a nosso ver, as classificações de Beck e de Haddad oferecem informações mais precisas e coerentes com os tipos de lesões acetabulares normalmente encontradas, e depende da familiaridade de cada cirurgião escolher entre uma delas na sua rotina.

$\mathrm{O}$ presente estudo apresenta algumas limitações. Por se tratar de um estudo retrospectivo, ele está sujeito a viés de seleção na escolha dos vídeos. Em segundo lugar, os observadores incluídos fazem parte de uma mesma equipe e participam de reuniões constantes para discussão do tema, o que pode ter aumentado a taxa de concordância entre os observadores. Por último, os vídeos foram editados para durar relativamente pouco tempo quando comparados com a duração total do procedimento cirúrgico, portanto os observadores obtiveram informações limitadas para classificar as lesões. Caso os observadores classificassem as lesões com base no vídeo completo da cirurgia, a presença de um maior número de informações poderia diminuir a reprodutibilidade encontrada.

\section{Conclusão}

Na nossa série, as classificações de Outerbridge, Beck e Haddad apresentaram boa reprodutibilidade interobservador e excelente reprodutibilidade intraobservador ao avaliar lesões condrais acetabulares por via artroscópica.

\section{Conflitos de Interesses}

Os autores declaram não haver conflitos de interesses.

\section{Referências}

1 de Amorim Cabrita HA, de Castro Trindade CA, de Campos Gurgel HM, Leal RD, de Souza Marques RdaF. Hip arthroscopy. Rev Bras Ortop 2014;50(03):245-253

2 Sampson TG. Arthroscopic treatment for chondral lesions of the hip. Clin Sports Med 2011;30(02):331-348

3 Amenabar T, Piriz J, Mella C, Hetaimish BM, O’Donnell J. Reliability of 3 different arthroscopic classifications for chondral damage of the acetabulum. Arthroscopy 2015;31(08):1492-1496

4 Ganz R, Parvizi J, Beck M, Leunig M, Nötzli H, Siebenrock KA. Femoroacetabular impingement: a cause for osteoarthritis of the hip. Clin Orthop Relat Res 2003;(417):112-120

5 Bedi A, Kelly BT. Femoroacetabular impingement. J Bone Joint Surg Am 2013;95(01):82-92

6 Kowalczuk M, Yeung M, Simunovic N, Ayeni OR. Does femoroacetabular impingement contribute to the development of hip osteoarthritis? A systematic review. Sports Med Arthrosc Rev 2015;23(04):174-179

7 Yeung M, Khan M, Schreiber VM, et al. Global discrepancies in the diagnosis, surgical management, and investigation of femoroacetabular impingement. Arthroscopy 2014;30(12):1625-1633

8 Oliveira FA, Basile R, Pereira BC, Cunha RL. Avaliação da reprodutibilidade da classificação de Tronzo para fraturas intertrocantéricas do fêmur. Rev Bras Ortop 2014;49(06):581-585

9 Tönnis D. Congenital dysplasia and dislocation of the hip in children and adults. New York: Springer-Verlag; 1987

10 Outerbridge RE. The etiology of chondromalacia patellae. J Bone Joint Surg Br 1961;43-B:752-757

11 Beck M, Kalhor M, Leunig M, Ganz R. Hip morphology influences the pattern of damage to the acetabular cartilage: femoroacetabular impingement as a cause of early osteoarthritis of the hip. J Bone Joint Surg Br 2005;87(07):1012-1018

12 Konan S, Rayan F, Meermans G, Witt J, Haddad FS. Validation of the classification system for acetabular chondral lesions identified at arthroscopy in patients with femoroacetabular impingement.J Bone Joint Surg Br 2011;93(03):332-336

13 Cohen J. A coeficiente of agrément for nominal scales. Educ Psychol Meas 1960;20(01):37-46

14 Landis JR, Koch GG. The measurement of observer agreement for categorical data. Biometrics 1977;33(01):159-174

15 Nepple JJ, Larson CM, Smith MV, et al. The reliability of arthroscopic classification of acetabular rim labrochondral disease. Am J Sports Med 2012;40(10):2224-2229

16 Lasmar NP, Lasmar RC, Vieira RB, de Oliveira JR, Scarpa AC. Assessment of the reproducibility of the Outerbridge and FSA classifications for chondral lesions of the knee. Rev Bras Ortop 2015;46(03):266-269

17 Cameron ML, Briggs KK, Steadman JR. Reproducibility and reliability of the outerbridge classification for grading chondral lesions of the knee arthroscopically. Am J Sports Med 2003;31(01):83-86 